\title{
Gene Expression Changes in a Model Neuron Cell Line Exposed to Autoantibodies from Patients with Traumatic Brain Injury and/ or Type 2 Diabetes
}

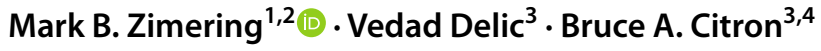 \\ Received: 16 March 2021 / Accepted: 11 May 2021 / Published online: 19 May 2021 \\ (c) This is a U.S. government work and not under copyright protection in the U.S.; foreign copyright protection may apply 2021
}

\begin{abstract}
Traumatic brain injury and adult type 2 diabetes mellitus are each associated with the late occurrence of accelerated cognitive decline and Parkinson's disease through unknown mechanisms. Previously, we reported increased circulating agonist autoantibodies targeting the 5-hydroxytryptamine 2A receptor in plasma from subsets of Parkinson's disease, dementia, and diabetic patients suffering with microvascular complications. Here, we use a model neuron, mouse neuroblastoma (N2A) cell line, to test messenger RNA expression changes following brief exposure to traumatic brain injury and/or type 2 diabetes mellitus plasma harboring agonist 5-hydroxytryptamine $2 \mathrm{~A}$ receptor autoantibodies. We now report involvement of the mitochondrial dysfunction pathway and Parkinson's disease pathways in autoantibody-induced gene expression changes occurring in neuroblastoma cells. Functional gene categories upregulated significantly included cell death, cytoskeletonmicrotubule function, actin polymerization or depolymerization, regulation of cell oxidative stress, mitochondrial function, immune function, protein metabolism, and vesicle function. Gene categories significantly downregulated included microtubule function, cell adhesion, neurotransmitter release, dopamine metabolism synaptic plasticity, maintenance of neuronal differentiation, mitochondrial function, and cell signaling. Taken together, these results suggest that agonist 5-hydroxytryptamine receptor autoantibodies (which increase in Parkinson's disease and other forms of neurodegeneration) mediate a coordinating program of gene expression changes in a model neuron which predispose to neuro-apoptosis and are linked to human neurodegenerative diseases pathways.
\end{abstract}

Keywords Neurodegeneration $\cdot$ Autoantibodies $\cdot$ Neurites $\cdot$ Cell death $\cdot$ RNAseq $\cdot$ Gene expression

\section{Introduction}

Traumatic brain injury (TBI) is a leading cause of long-term disability in US military combat veterans. Neuropsychiatric disorders and cognitive decline are "early" and "late"

Mark B. Zimering

mark.zimering@va.gov

1 Endocrine and Diabetes Section, Medical Service, VA New Jersey Healthcare System, 385 Tremont Ave, East Orange, NJ 07018, USA

2 Robert Wood Johnson Medical School, New Brunswick, NJ, USA

3 Laboratory of Molecular Biology, VA New Jersey Health Care System, Research \& Development (Mailstop 15), 385 Tremont Ave, East Orange, NJ 07018, USA

4 Pharmacology, Physiology and Neuroscience, New Jersey Medical School, Rutgers University, Newark, NJ, USA neurologic manifestations of exposure to "blast" and direct force brain injuries [1,2]. While consequences of TBI have been linked to etiology of several neurodegenerative diseases, the most compelling evidence currently available is for a biological link between TBI and Parkinson's disease (PD) [3]. PD is the most common of all the parkinsonian diseases which also include corticobasal degeneration syndrome, Dementia with Lewy bodies, multisystem atrophy, progressive supranuclear palsy, and vascular parkinsonism. These diseases are grouped together based on clinical presentation and pathological similarities which includes progressive neurodegeneration [4].

Vascular injury and inflammation may have an etiologic role in neuropsychiatric and neurodegenerative complications following TBI [5] in part via activation of humoral immune responses to antigens released following injury. One such putative antigen, the 5-hydroxytryptamine 2 a receptor (5-HT2AR) is widely distributed 
in the central nervous system where it functions in the regulation of mood, thinking, perception, memory, sleep, and other important functions [6]. 5-HT2AR is a subtype of the 5-HT2R, a member of the G protein-coupled family of serotonin receptors [7]. Activation of 5-HT2AR is necessary for hallucinations associated which psychedelic drugs such as LSD which act as full or partial 5-HT2AR agonists [8]. In a recent report, long-acting agonist autoantibodies to the 5-HT2AR were increased in veterans suffering TBI in association with the late occurrence of neurodegenerative complications, e.g., dementia, Parkinson's disease [9]. The long-acting agonist antibody to 5-HT2A receptor (in adult TBI, and type 2 diabetes mellitus without TBI) caused acute neurite retraction and accelerated cell loss in mouse neuroblastoma N2A cells via inositol triphosphate receptor-mediated cytosolic $\mathrm{Ca}^{2+}$ signaling $[9,10]$. Normal $\mathrm{Ca}^{2+}$ is essential for helping orchestrate neuronal gene expression during development, as well as to maintain homeostasis which is altered following a TBI [11]. Neurons respond to insult and environmental stimuli by modulating $\mathrm{Ca}^{2+}$ release and reuptake from two major intracellular calcium stores endoplasmic reticulum and mitochondria [12-14]. Abnormal $\mathrm{Ca}^{2+}$ signaling may in turn lead up to gene expression changes that cause or contribute to buildup of toxic proteins (e.g., alpha synuclein, amyloid beta, Tau) and cause mitochondrial dysfunction resulting in neurodegeneration and cell death $[3,15]$.

Type 2 diabetes mellitus increases in aging veterans. We compared messenger RNA expression occurring downstream of 5-HT2A receptor activation in neuronal cells exposed to agonist 5-HT2AR autoantibodies isolated from plasma of traumatic brain injury patients with or without co-morbid type 2 diabetes and in type 2 diabetes without traumatic brain injury.

\section{Materials and Methods}

Participants Participants were enrolled in local Veteran Affairs New Jersey Health Care System IRB-approved studies of the interaction between type 2 diabetes and traumatic brain injury in the late occurrence of neurodegeneration. All participants signed IRB-approved consent prior to blood drawing for autoantibodies isolation. Plasma was obtained in the morning.

All participants are males who suffered direct force head trauma. The time interval following TBI was variable ranging from 1 to 20 years or longer. Nearly all of the TBI patients ( 5 of 6 ) suffered a single, direct force mild TBI, i.e., concussion. None of the participants experienced blast injury. One of six TBI patients had experienced multiple mild TBIs which is expected to be associated with higher level and titer of harm-inducing autoantibodies [9]. The TBI patients were not selected on the basis of having had exposure to a particular type of TBI. The TBI patients were selected on the basis of harboring plasma 5-HT2AR binding autoantibodies that displayed 2.8-fold or higher binding ( $v s$. background) in the ELISA. They were representative of men exposed to the most common type of TBI - a single mild direct force TBI [16] All diabetes patients had type 2 diabetes mellitus, and they were treated with a number of different oral anti-diabetic medications and/or insulin or incretinbased therapies.

Subgroups Each of four subgroups of participants was represented by three patients:

diabetes without harm-inducing plasma autoantibodies; diabetes harboring harm-inducing plasma autoantibodies without TBI, TBI alone with harm-inducing autoantibodies; and TBI+diabetes with harm-inducing autoantibodies.

Protein-A Affinity Chromatography Protein A affinity chromatography was performed as previously reported [17].

Enzyme-Linked Immunoassay for 5-HT2A Receptor The 5-HT2AR binding assay was carried out as previously reported [18] using (as capture antigen) an 18-meric linear synthetic peptide, Q....N, (LifeTein Inc., Hillsborough, $\mathrm{NJ}$ ) having amino acid sequence identical to that of the second extracellular loop region of the human 5-HT2AR. A $1 / 25$ th dilution of the protein-A eluate fraction of plasmas was added to each well in duplicate. Autoantibody-antigen binding was detected with the use of peroxidase conjugated, goat-anti-human IgG (Sigma Chem Co, St Louis, MO) and appropriate substrate solution [18]. Color development was monitored at $490 \mathrm{~nm}$ using an IMark reader (Biorad).

Cells Mouse neuroblastoma N2A cells were obtained from American Type Culture Collection (Manassa, VA). Mouse neuroblastoma cells (N2A) were maintained in Dulbecco's modified Eagle's medium containing 10\% fetal bovine serum. An identical number of cells was seeded into each of twelve T- $75 \mathrm{~cm}^{2}$ flasks and incubated for $48 \mathrm{~h}$ until the cells had reached $\sim 80 \%$ confluency. They were seeded at $2 \times 10$ ${ }^{6}$ cells/ T-75 $\mathrm{cm}^{2}$ flask, and after 3 passages in culture $(1: 3$ subcultivation ratio), each of twelve $80 \%$ confluent $\mathrm{T}-75 \mathrm{~cm}^{2}$ flasks contained $\sim 5.6 \times 10^{6}$ cells. Next a 1:25th dilution of each of the twelve protein A-eluate fractions of plasma was added to each flask of N2A cells (1 patient autoantibody/ flask). The cells were incubated (in the presence of autoantibody) for $2 \mathrm{~h}$ at $37^{\circ} \mathrm{C}$ prior to RNA extraction.

Neurites The N2A neurite retraction assay was performed as previously reported [17]. Briefly, mouse neuroblastoma 
cells were seeded at low density in $35 \mathrm{~mm}$ dishes. After $24 \mathrm{~h}$ incubation to allow cell attachment and spontaneous neurite expression, a test protein-A eluate fraction of patient plasma was added (1:25 dilution) to dishes. Cells were observed over a 10-min time period for acute change in baseline neurite length. Percent basal neurite length represents the average of the (autoantibody stimulated/basal neurite length) in ten or more cells from two dishes, each cell expressing two or more neurites.

Cell Survival The measurement of accelerated mouse neuroblastoma cell loss was performed as previously reported [17]. Cell survival of N2A cells incubated for $(12-16 \mathrm{~h})$ with a $1: 50$ th dilution of the protein-A eluate fraction of plasma was estimated using an MTT assay. Proportion of surviving cells represents the average of quadruplicate determinations compared to basal cell number in wells not containing test protein-A eluate fractions.

RNA Analysis RNA extraction was performed using the Rneasy Plus Mini Kit (QIAGEN cat. 74,134), according to the manufacturer's specifications, on approximately 1 million N2A cells per flask.

For RNAseq, RNA samples were provided to the Rutgers Genomics Center, and they returned the sequences obtained. Briefly, the RNA integrity numbers were confirmed as greater than 7.0 with an Agilent TapeStation 2200, and poly(A) mRNAs were selected from the total RNA samples with oligo-d(T) 25 magnetic beads (New England Biolabs). cDNA libraries were generated with the NEB next ultra RNAseq library kit and purified with AmpureXP beads and further characterized with an Agilent TapeStation and fluorescence (qubit) analysis. These libraries, specifically barcoded for each sample, were pooled in equimolar amounts and sequence for a $1 \times 75$ base read configuration with a NextSeq 500 instrument (Illumina, San Diego, CA). An average of 3.5 Gbases of sequence information was obtained per sample (corresponding to approximately 47 million sequences per sample).

Pathway Analysis CLC Genomics Workbench version 20.0, QIAGEN (Germantown, MD) was used to map the sequences obtained to the mouse genome and perform initial expression analyses. Ingenuity Pathway Analysis, QIAGEN (Germantown, MD) was used to identify pathways associated with genes dysregulated by patient autoantibodies.

Statistical Analysis Mean values are depicted \pm standard deviation and were compared by ANOVA and Bonferroni post-hoc testing with $\mathrm{P}<0.05$ indicating significance.

\section{Results}

\section{Autoantibodies in Subsets of TBI, T2DM and $T B I+T 2 D M$}

Three subgroups of "test" patients were selected on the basis of having diagnoses of TBI with or without type 2 diabetes mellitus (T2DM) or T2DM without TBI $(n=3$ in each subgroup) and who also harbored plasma autoantibodies which demonstrated high level of binding (3-3.5-fold background) to a linear synthetic peptide corresponding to the second extracellular loop of the human 5-HT2A receptor (Table 1). The control group consisted of age-matched patients with T2DM and without TBI $(n=3)$ whose plasma autoantibodies lacked significant binding to the 5-HT2A receptor linear synthetic peptide (Table 1). The incidence of late-onset sporadic form of PD increases in older adults with type 2 diabetes and following traumatic brain injury. Because of a previous association between PD and high level of autoantibody binding to the 5-HT2A receptor peptide [18], in the current study "test" patients were enriched by inclusion of $4 / 9$ patients having PD vs. $0 / 3$ "control" patients with PD (Table 1). Gene expression changes in response to 2-h incubation of mouse neuroblastoma (N2A) cells with a 1:25 dilution of patient autoantibodies was compared in "test" $(n=9)$ vs "control" $(n=3)$ patients who differed significantly in baseline autoantibody binding to the 5-HT2A receptor peptide.

\section{Effect of Diabetic and/or TBI Plasma Autoantibodies on Neuroblastoma Neurite Retraction or Cell Survival}

As previously reported [18], autoantibodies in subsets of older adult type $2 \mathrm{DM}$, adult TBI, or T2DM + TBI which displayed increased binding to a linear synthetic peptide corresponding to the second extracellular loop domain of the human 5-HT2AR were neurotoxic. The length of the processes extended by the model neurons is an indicator of

Table 1 Baseline characteristics of the study participants

\begin{tabular}{lccll}
\hline Group & N & Age (years) & $\begin{array}{l}\text { 5-HT2AR } \\
\text { binding (AU) }\end{array}$ & No. with PD \\
\hline Non-toxic DM & 3 & $74.7 \pm 9.0$ & $0.06 \pm 0.02$ & 0 \\
TBI & 3 & $70.7 \pm 6.1$ & $0.14 \pm 0.06$ & 1 \\
DM & 3 & $62.0 \pm 7.0$ & $0.18 \pm 0.03$ & 2 \\
TBI+DM & 3 & $67.7 \pm 8.1$ & $0.15 \pm 0.02$ & 1 \\
\hline
\end{tabular}

Results are mean $\pm \mathrm{SD}$; $\mathrm{AU}=$ arbitrary absorbance units $(0.05$ is the background level) in the ELISA employing a linear synthetic 18-meric peptide corresponding to the second extracellular loop of the 5-HT2AR receptor 
cellular health. As shown in Fig. 1a, exposure to the neurodegenerative autoantibodies caused significantly greater mean acute neurite length-shortening (i.e., retraction) in N2A mouse neuroblastoma cell vs nontoxic DM autoantibodies $(\mathrm{P}=0.01)$ (Fig. 1a).

Cell loss was also monitored after incubation with the autoantibodies. The toxic autoantibody treatments promoted significantly greater mean accelerated loss in N2A neuroblastoma cells vs nontoxic DM autoantibodies $(\mathrm{P}=0.001)$ (Fig. 1b).

\section{Gene Expression Changes in TBI + DM vs. Control Antibodies}

A volcano plot (Fig. 2) illustrates the genes that displayed an up (red) or down (green) regulation in excess of 1.5-fold and a statistically significant $p$ value in response to $T B I+D M$ antibodies vs. control antibodies. A larger number of genes was significantly upregulated compared to genes significantly downregulated.

\section{Expression of Genes Affected by the Neurodegenerative Autoantibodies}

Figure 3 depicts the fold changes induced by the autoantibodies from all three conditions with the $\mathrm{x}$-axis representing fold changes from the TBI patient samples, the y-axis indicating the fold changes from the DM patient samples, and the color indicating the fold changes induced by the samples from the patients with TBI history and diabetes. This graph includes any gene where the expression levels from any condition met the expression cutoff of $50 \mathrm{rpkm}$ and where the fold change for any sample group had a p value $<0.05$. One can see that, with only some exceptions, any of the conditions that were experimentally found to negatively impact the health of the N2a model neurons produced relatively

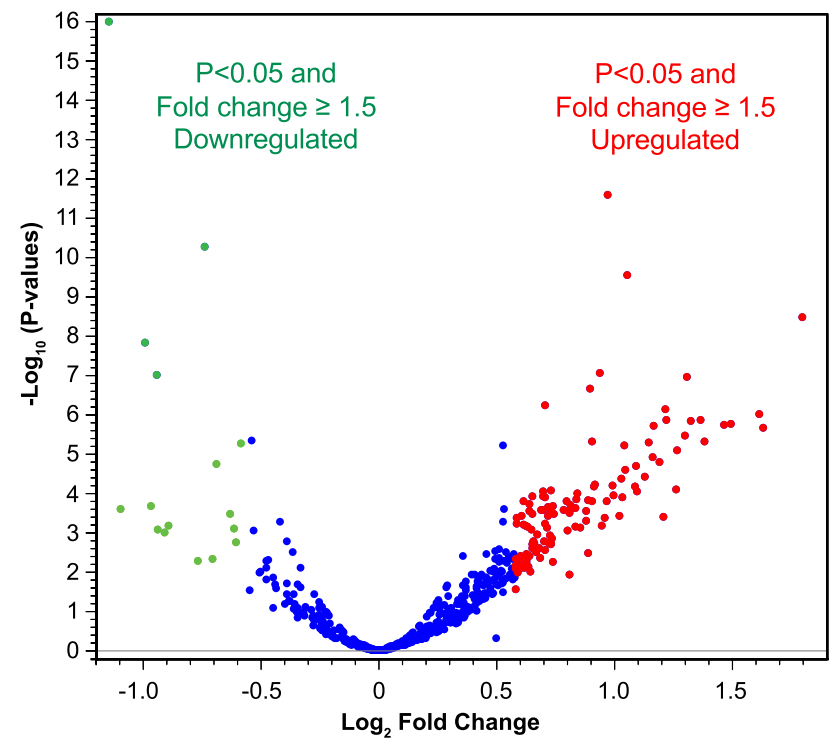

Fig. 2 Statistical analysis of dysregulated genes. Gene expression changes in response to TBI + Db antibodies compared to control antibodies display mostly upregulated genes. A volcano plot illustrates the genes that displayed up (red) or down (green) regulation in excess of 1.5 -fold and a statistically significant $\mathrm{p}$ value

similar gene changes among the 25,000 mRNA sequences that were examined.

\section{Genes Up- or Downregulated by Autoantibodies in Plasma from TBI and/or DM Autoantibodies}

Table 2 lists the genes significantly dysregulated by exposure to plasma autoantibodies from TBI and/or T2DM patients. Among the individual genes upregulated by autoantibodies in TBI and/or T2DM plasma are those encoding proteins having a role in ribosomal structure and function (RP19-ps6, Rps14), programmed cell death
Fig. 1 The effects of autoantibodies from TBI and type 2 diabetic patients on neuroblastoma cell health. a Acute neurite retraction of $\mathrm{N} 2 \mathrm{~A}$ model neurons was induced by autoantibodies from TBI and DM subsets. b Accelerated N2A cell loss from the autoantibodies. $\left(\mathrm{N}=3\right.$ per group, ${ }^{*} \mathrm{p}<0.01$, $* * \mathrm{p}<0.001$ vs. the nontoxic control antibodies) a

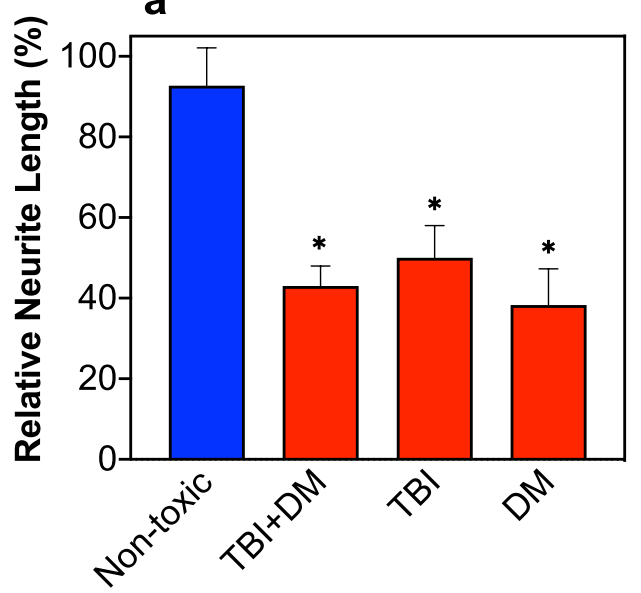

Autoantibodies b

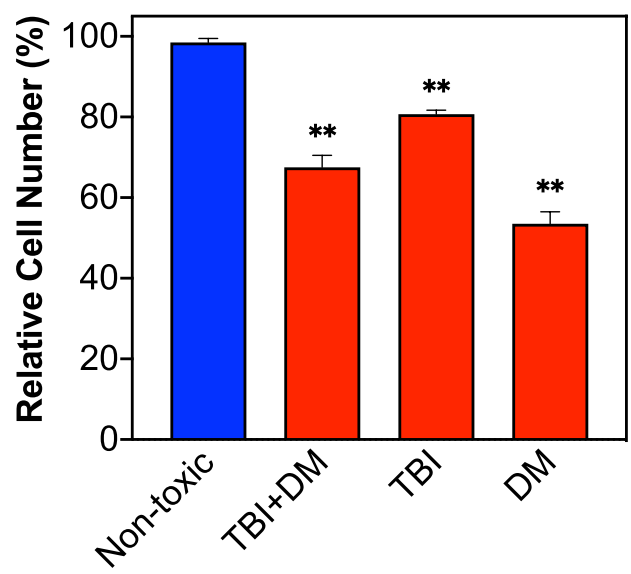

Autoantibodies 


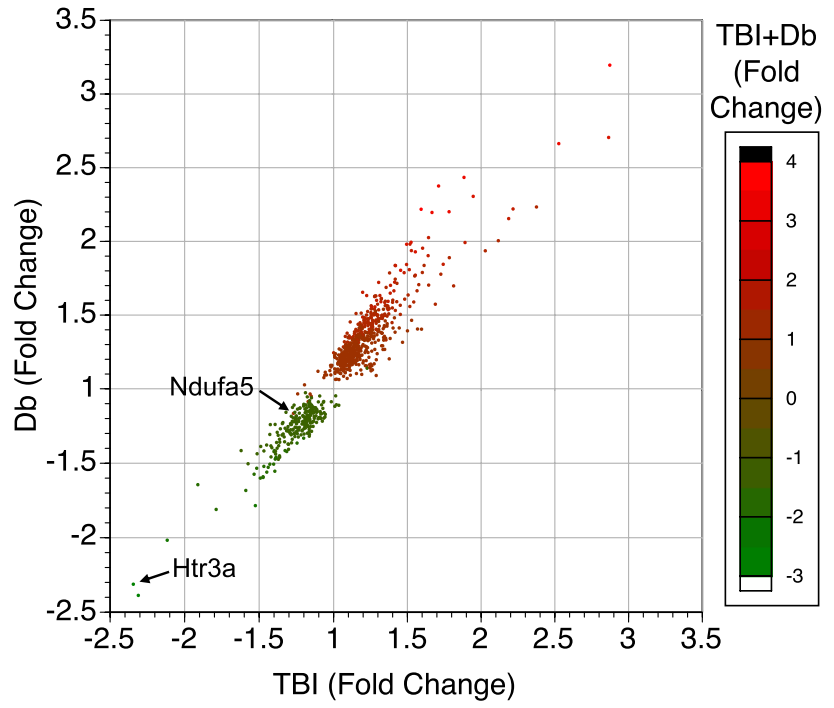

Fig. 3 Expression of genes affected by the neurodegenerative autoantibodies. RNAseq mRNA values are shown for 806 genes and indicate gene expression fold changes of all of the test groups. Fold changes induced by the toxic TBI autoantibodies (x-axis), toxic $\mathrm{Db}$ autoantibodies (y-axis) and the $\mathrm{TBI}+\mathrm{Db}$ autoantibodies (color scale). Each dot's position and color represents the fold change seen relative to exposure to the control, non-toxic autoantibody samples. Some genes displayed discordance, e.g., Ndufa5 (NADH:ubiquinone oxidoreductase subunit A5) was downregulated $15-25 \%$ by TBI or Db samples, but was not downregulated by the $\mathrm{TBI}+\mathrm{Db}$ samples and appears red. Htr3a was one of the most downregulated genes by autoantibodies from all 3 toxic conditions

(Pdcd10), cytotoxic T lymphocyte regulation (Ctla2a), tumor suppression(Rassf1), zinc finger encoded proteins (Znhit3), enzymatic suppression of apoptosis in resting lymphocytes (Dpp7), and B lymphocyte differentiation (Xlr3a) (Table 2). Functional categories comprising several or more individual genes that were upregulated significantly include: cell death, cholesterol biosynthesis, cytoskeleton-microtubule function and actin polymerization or depolymerization, regulation of cell oxidative stress, mitochondrial function, immune function, protein metabolism, and vesicle function (Table 2). Individual genes significantly downregulated by exposure to autoantibodies in TBI and/or T2DM include those having a role in microtubule function (MAP1B), cell adhesion (Nectin1, Ncam1, L1 cam), neurotransmitter release or dopamine metabolism (synapsin II, complexin I, dopamine beta hydroxylase), flow-sensing in aortic endothelium (Gpr68), synaptic plasticity underlying learning and memory (Arc), maintenance of neuronal differentiation (nerve growth factor receptor), at least six genes involved in mitochondrial function (e.g., cytochrome b, and NADH dehydrogenase, subunit 4L (complex I)), and several genes (including G-protein coupled receptors) having a role in cell signaling: 5-hydroxytryptamine 3a receptor, G-protein coupled receptor kinase 3 (GRK3), and glypican 1- a cell surface heparan sulfate proteoglycan (Table 2).

\section{Effect of TBI and/or DM Autoantibodies on Gene Expression in Serotonergic Receptors}

The mammalian 5-HT2A receptor is highly expressed in brain regions important in the regulation of mood, thinking, perception, sleep, and feeding behaviors [6]. In many of the same brain regions, other members of the 5-HT receptor family (total 14 isoforms) are also expressed and may have coordinate or antagonistic functions as those of the 5-HT2A receptor. Of interest, only the Htr2a and Htr3a genes were significantly expressed (i.e., RPKM $>50$ ) in N2A mouse neuroblastoma cells, and expression of $\mathrm{Htr} 2 \mathrm{a}$ was not altered following exposure to TBI and/or DM autoantibodies. The $\mathrm{Htr} 3 \mathrm{a}$ gene displayed significant downregulation across all three test subgroups vs control, and a significant $p$ value (Table 2). Htr3A is the only member of the 5-HT receptor family which encodes an ion channel [19]. 5htr3a co-localizes with AchR on presynaptic terminals in the striatum [20]. The significant downregulation of $5 \mathrm{htr} 3 \mathrm{a}$ by the test group patient autoantibodies vs control is interesting since 5htr3a normally functions to increase dopamine neurotransmitter release in the striatum [20]. Thus, decreased expression of the 5htr3a receptor (in response to TBI and T2DM autoantibodies) may interfere with dopamine release in the striatuma hallmark of Parkinson's disease.

A striking feature of the autoantibodies effect on serotonin $2 \mathrm{~A}$ receptor-mediated neurite retraction in neuroblastoma cells was the previously reported finding [17] that the phenotypic response did not undergo "rapid desensitization" which is typical of G-protein coupled receptor signaling. Of interest, expression of G-protein receptor kinase 3 (Grk3) which normally functions in mediating desensitization of G-protein coupled signaling [21] was significantly downregulated (in N2A cells) by the TBI and/or T2DM autoantibodies.

\section{Gene Ontology Analysis}

Gene ontology revealed several processes underlying the gene expression changes in response to autoantibodies in TBI and/ or T2DM. First, the "neuron apoptosis process" included four genes which were significantly upregulated in N2a cells treated with test patient autoantibodies including Casp3, Bnip3, Nae1, and Gpx1. In a prior report, diabetic plasma autoantibodies in patients having microvascular complications caused endothelial cell apoptosis via activation of caspase 3 [22], an important mediator of apoptosis. Bnip3 is a member of the apoptotic Bcl-2 protein family which plays a role in permeabilization of the outer mitochondrial membrane. NEDD8 activating enzyme E1 subunit 1 (product of the Nae1 gene) is involved 
Table 2 Genes dysregulated by exposure to autoantibodies affecting cellular health

\begin{tabular}{|c|c|c|c|c|}
\hline Change direction & Fold changevs. control & & & \\
\hline \multicolumn{5}{|l|}{ Category } \\
\hline Gene symbol & Entrez gene name & TBI & $\mathrm{DM}$ & TBI/DM \\
\hline \multicolumn{5}{|l|}{ Genes upregulated } \\
\hline \multicolumn{5}{|l|}{ Cell death } \\
\hline DDIT4 & DNA damage inducible transcript 4 & 2.12 & 2.01 & 1.62 \\
\hline Pdcd 10 & Programmed cell death protein 10 & 1.85 & 2.3 & 3.23 \\
\hline Casp3 & Caspase 3 & 1.27 & 1.34 & 1.3 \\
\hline Bnip3 & $\mathrm{Bcl} 2$ interacting protein 3 & 1.56 & 1.98 & 2.69 \\
\hline Nae1 & NEDD8 activating enzyme E1 subunit 1 & NS & 1.43 & 1.52 \\
\hline \multicolumn{5}{|c|}{ Cholesterol biosynthesis } \\
\hline MSMO1 & Methyl sterol monooxygenase 1 & 2.87 & 3.2 & 3.64 \\
\hline MVD & Mevalonate pyrophosphate decarboxylase & 2.86 & 2.71 & 2.63 \\
\hline Cyp51 & Cytochrome p450, family 51 & 1.89 & 1.99 & 2.3 \\
\hline Hmgcs1 & 3-hydroxy-3-methylglutaryl-CoA synthase & 2.22 & 2.22 & 2.3 \\
\hline ACAT2 & acetyl-CoA acetyltransferase 2 & 2.19 & 2.15 & 2.08 \\
\hline Insig 1 & insulin-induced gene 1 & 1.74 & 1.84 & 2.08 \\
\hline NSDHL & $\mathrm{NAD}(\mathrm{P})$ dependent steroid dehydrogenase-like & 2.1 & 2.15 & 2.03 \\
\hline \multicolumn{5}{|l|}{ Cytoskeleton } \\
\hline NME2 & Nucleoside diphosphate kinase 2 & 1.78 & 2.2 & 3.07 \\
\hline TPPP3 & Tubulin polymerization promoting protein family member 3 & 1.6 & 1.79 & 1.87 \\
\hline TUBA1A & Tubulin alpha $1 \mathrm{a}$ & 1.78 & 1.89 & 1.79 \\
\hline BRK1 & BRICK1 subunit of SCAR/WAVE actin nucleating complex & NS & 1.41 & 1.45 \\
\hline \multicolumn{5}{|l|}{ Cell oxidation } \\
\hline GPX4 & Glutathione peroxidase 4 & 1.55 & 1.77 & 1.87 \\
\hline GPX1 & Glutathione peroxidase 1 & 1.52 & 1.56 & 1.57 \\
\hline PARK7 & Parkinsonism associated deglycase & NS & NS & 1.34 \\
\hline \multicolumn{5}{|c|}{ Signaling/signal transduction } \\
\hline KLF6 & Kruppel like factor 6 transcription factor & 2.37 & 2.12 & 2.22 \\
\hline PPP1R35 & Protein phosphatase 1 regulatory subunit 35 & 1.69 & 1.99 & 2.09 \\
\hline BEX4 & Brain expressed X-linked 4 & 1.65 & 1.66 & 2.06 \\
\hline TRIB3 & Tribbles pseudokinase 3 & 2.17 & 1.91 & 1.95 \\
\hline GDF15 & Growth diff factor 15 & 1.93 & 1.72 & 1.72 \\
\hline CD63 & Cd63 antigen & 1.51 & 1.64 & 1.66 \\
\hline RABAC1 & Rab acceptor 1 & 1.45 & 1.57 & 1.63 \\
\hline RACK1 & Receptor for activated $\mathrm{C}$ kinase 1 & 1.37 & 1.56 & 1.65 \\
\hline \multicolumn{5}{|c|}{ Mitochondrial function } \\
\hline Ndufb8 & NADH dehydrogenase 1 beta subunit 8 & 1.53 & 1.94 & 2.25 \\
\hline Ndufaf6 & NDH: ubiquinone oxidoreductase complex & 1.51 & 1.98 & 2.2 \\
\hline Fmc1 & Formation of mitochondrial complex $\mathrm{V}$ assembly factor 1 homolog & 1.51 & 1.81 & 2.01 \\
\hline Atp5e & ATP synthase, $\mathrm{H}+$ transporting mitochondrial $\mathrm{F} 1$ complex, epsilon subunit & 1.41 & 1.72 & 2.32 \\
\hline Apt5mg & ATP synthase membrane subunit $g$ & 1.37 & 1.54 & 2.12 \\
\hline \multicolumn{5}{|l|}{ Immune function } \\
\hline BRI3 & Brain protein $\mathrm{I} 3$ & 1.56 & 1.79 & 1.94 \\
\hline CTL2a & Cytotoxic T lymphocyte associated protein 2 alpha & 2 & 2.25 & 2.91 \\
\hline CREG1 & Cellular repressor of E1A stimulated genes 1 & 1.68 & 1.76 & 1.84 \\
\hline \multicolumn{5}{|c|}{ Protein metabolism } \\
\hline TOMM5 & Translocase of outer mitochondrial membrane & 2.03 & 1.64 & 2.46 \\
\hline PSMA6 & Proteasome 20 S subunit alpha 6 & 1.39 & 1.7 & 2.13 \\
\hline \multicolumn{5}{|l|}{ Vesicle function } \\
\hline NAP1L5 & NSF attachment protein alpha & 1.63 & 1.62 & 1.51 \\
\hline
\end{tabular}


Table 2 (continued)

\begin{tabular}{|c|c|c|c|c|}
\hline Change direction & Fold changevs. control & & & \\
\hline \multicolumn{5}{|c|}{ Genes downregulated } \\
\hline \multicolumn{5}{|c|}{ Signaling/signal transduction } \\
\hline HTR3A & 5-hydroxytryptamine $3 \mathrm{~A}$ receptor & -2.34 & -2.31 & -2.73 \\
\hline RET & Ret proto-oncogene & -1.79 & -1.81 & -1.92 \\
\hline GPC1 & Glypican & -1.52 & -1.53 & -1.69 \\
\hline SRF & Serum response factor & -1.69 & -1.59 & -1.63 \\
\hline GPR69 & G protein coupled receptor 68 & -1.52 & -1.63 & -1.76 \\
\hline GRK3 & $\mathrm{G}$ protein coupled receptor kinase 3 & -1.59 & -1.66 & -1.95 \\
\hline \multicolumn{5}{|l|}{ Cytoskeleton } \\
\hline TLN2 & Talin 2 & -1.64 & -1.7 & -1.74 \\
\hline MAP1B & Microtubule associated protein $1 \mathrm{~B}$ & -1.4 & -1.52 & -1.51 \\
\hline ACTG1 & Actin gamma 1 & -1.47 & -1.38 & -1.42 \\
\hline \multicolumn{5}{|c|}{ Extracellular matrix } \\
\hline NCAM1 & Neural cell adhesion molecule 1 & -1.71 & -1.7 & -1.71 \\
\hline L1CAM & L1 cell adhesion molecule & -1.58 & -1.66 & -1.71 \\
\hline Nectin 1 & Nectin cell adhesion molecule 1 & -1.76 & -1.79 & -1.77 \\
\hline \multicolumn{5}{|c|}{ Neurotransmitter release/synthesis } \\
\hline SYN2 & Synapsin II & -1.56 & -1.71 & -2.23 \\
\hline $\mathrm{DBH}$ & Dopamine beta hydroxylase & -2.31 & -2.39 & -2.69 \\
\hline CPLX1 & Complexin I & -1.43 & -1.51 & -1.67 \\
\hline \multicolumn{5}{|c|}{ Neuronal differentiation } \\
\hline PLXNA4 & PlexinA4 & -1.65 & -2.07 & -2.7 \\
\hline NGFR & Nerve growth factor receptor & -1.39 & -1.47 & -1.61 \\
\hline \multicolumn{5}{|c|}{ Mitochondrial function } \\
\hline MT-ND4L & NADH dehydrogenase, subunit 4L (complex I) & -1.53 & -1.79 & -2.13 \\
\hline MT-ND4L & NADH dehydrogenase, subunit 4 (complex I) & -1.47 & -1.59 & -1.91 \\
\hline MT-ND5 & NADH dehydrogenase, subunit 5 (complex I) & -1.44 & -1.53 & -1.87 \\
\hline MT-CYB & Cytochrome B & -1.45 & -1.56 & -1.85 \\
\hline MT-ND6 & NADH dehydrogenase, subunit 6 (complex I) & -1.4 & -1.42 & -1.63 \\
\hline MT-ND1 & NADH dehydrogenase, subunit 1 (complex I) & NS & NS & -1.46 \\
\hline
\end{tabular}

Upregulated and downregulated genes after exposure to autoantibodies from patients with traumatic brain injury and/or type 2 diabetes. NS not significant, i.e., $\mathrm{p} \geq 0.05$ vs. control

in neddylation - a process in which the ubiquitin-like protein NEDD8 is covalently bound to proteins. Neddylation has been implicated in Alzheimer's dementia by promoting neuron apoptosis via promotion of cell cycle entry [23]. Taken together, accelerated neuroblastoma cell loss following exposure to autoantibodies in diabetes, TBI and TBI plus DM from patients having co-morbid Parkinson's disease, or dementia [10] may be mediated by neuroapoptosis involving (in part) increased Casp3, Bnip3 and Nae1 gene expression. Glutathione peroxidase $1(\mathrm{Gpx} 1)$ protects the cell from oxidative damage. Significant upregulation of gene expression in Gpx1 and in proteasome subunits alpha 1 (Psma1) and beta type 4 (PSMB4) suggests likely involvement of the "negative regulation of inflammatory response to antigenic stimulus' process. The "response to nerve growth factor" process is suggested by significantly decreased expression of complexin-2 (Cplx2)
- a cytosolic protein involved in synaptic vesicle exocytosis. Decreased expression of Cplx2 is associated with schizophrenia and Huntington's disease [24] and with decreased neurotransmitter release [25]. Finally, significant upregulation of the gene $\mathrm{Zfand} 2 \mathrm{~b}$ which encodes a zinc finger type protein suggests additional involvement of the "regulation of insulin like growth factor receptor signaling pathway." Zfand $2 b$ protein product interacts with the proteasome in mediating proteostasis via binding to and helping to eliminate misfolded proteins from the cell.

\section{Signaling Pathways Affected by Degenerative Autoantibodies}

Compared to control exposures, the harm-inducing autoantibodies caused changes in several pathways. Focusing on 
the changes induced by TBI + Db subgroup autoantibodies, pathways that seemed biologically relevant included mitochondrial dysfunction pathway and Parkinson's disease pathway. Upstream regulators that hit statistically and seemed biologically interesting included NFE2L2 (Nrf2) upstream network. Of note, the Parkinson's disease pathway includes dysregulation of caspase 3 and Park 7 - two genes which were significantly upregulated following exposure to TBI + DM autoantibodies (Table 2) plus some genes that did not meet all criteria but were identified as statistical hits in the pathway analysis. These included upregulation of UCHL1 (involved in Lewy body formation) and Cyc5 (can activate caspase cascades). In this pathway, the only discrepancy was p38 MAPK that can upregulate Cyc5 and was expected to be upregulated; however it was found downregulated. Genetic factors which block dopamine release lead to dopamine accumulation and the production of reactive oxygen species, a component of the Parkinson's disease pathway. Three genes significantly downregulated by harm-inducing autoantibodies (synapsin II, complexin I, and htr3a) mediate neurotransmitter release, and a fourth downregulated gene, dopamine beta hydroxylase, metabolizes dopamine to norepinephrine. Taken together, their combined significant downregulation may contribute to dopamine accumulation. Dopamine is normally metabolized to H202 which inhibits mitochondrial complex I function - a key target in the mitochondrial dysfunction pathway. Evidence in favor of involvement of the mitochondrial dysfunction pathway is significant downregulation of five genes belonging to mitochondrial complex I by the harm-inducing autoantibodies (Table 2). Finally, a number of different genes significantly dysregulated by the harminducing autoantibodies provide linkage(s) to the Nrf2 upstream network including Syn2, Rack1, BNIP3, CREG1, Gpx1, L1CAM, ACTG1, PSMA6, and other genes encoding subunits of the proteasome (Table 2).

\section{Overlapping Effects of Autoantibodies from Different Neurodegenerative Conditions}

The Venn diagram indicates genes significantly up- or downregulated by TBI and T2DM 5-HT2AR binding plasma autoantibodies (Fig. 4). There was significant overlap in gene dysregulation among the three subgroups vs control. For example, a total of 99 genes were significantly upregulated in all three "test" subgroups compared to control (Fig. 4a). An additional 160 genes were significantly upregulated in both $\mathrm{TBI}+\mathrm{Db}$ and $\mathrm{Db}$ subgroups, but not in TBI alone. In contrast, only 2 genes were upregulated in both TBI $+\mathrm{Db}$ and TBI, but not in Db (Fig. 4a). TBI + Db had the highest number of significantly upregulated genes (395) followed by $\mathrm{Db}$ alone (268) and TBI alone (113). Total 64 genes were significantly downregulated in $\mathrm{TBI}+\mathrm{Db}$ antibodies a Upregulated genes.

TBI vs. Control

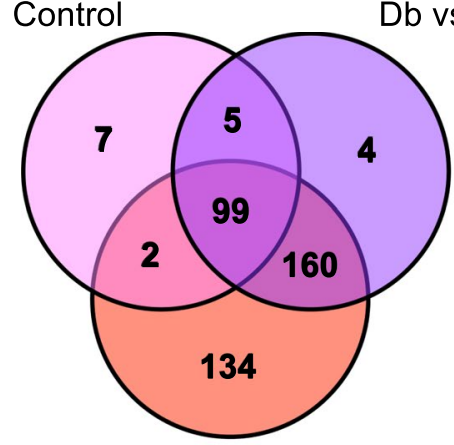

$\mathrm{TBI}+\mathrm{Db}$ vs. Control

\section{b Downregulated genes.}

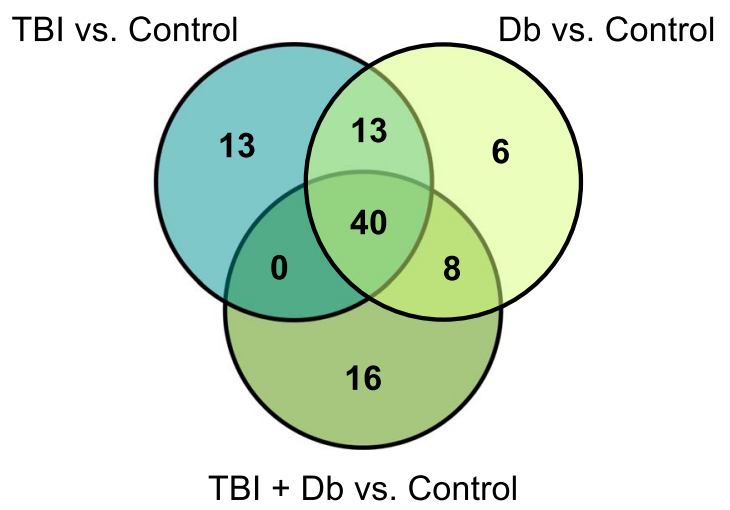

Fig. 4 Overlapping effects of autoantibodies from different neurodegenerative conditions. The Venn diagram indicates genes significantly up- (a) or downregulated (b) by TBI and T2DM 5-HT2AR binding plasma autoantibodies

compared to 66 genes in TBI and 67 genes in Db (Fig. 4b). Forty genes were significantly downregulated in all three subgroups vs control (Fig. 4b).

\section{Mitochondrial and Cell Oxidation Gene Expression Changes}

Genes associated with cell oxidation were found to increase in excess of 1.5 fold in TBI, DM and TBI/DM, with TBI/ DM generally having slightly higher expression fold change among cell oxidation genes for glutathione peroxidase 4 and 1. Parkinsonism associated deglycase gene was the only cell oxidation gene expression significantly elevated (1.34-fold) in TBI/DM group compared to control.

In general, genes corresponding to the mitochondrial electron transport chain complex I subunits were found to decrease, while subunits corresponding to mitochondrial complex V (ATP synthase) were found to be upregulated. Both proportion of up- and downregulation followed an increasing trend with TBI/DM having greatest increase 
followed by DM and TBI groups compared to controls. Translocase of outer mitochondrial membrane (TOMM5) was also found to increase 2.46-fold in TBI/DM, 2.03-fold in TIB, and 1.64-fold in DM groups compared to controls.

\section{Discussion}

These are the first data suggesting that long-lasting agonist 5-HT2A receptor-targeting autoantibodies in plasma from patients having TBI and/or T2DM and suffering with microvascular and neurodegenerative (e.g., PD) complications cause significant modulation of expression in genes underlying neuroapoptosis, the maintenance of differentiated neuronal morphology, neurotransmitter release, synaptic plasticity, and amyloid beta precursor protein interactions. The present findings are consistent with our prior reports that serotonin-2A receptor binding autoantibodies prevalent in $85 \%(17 / 20)$ of older adults with sporadic Parkinson's disease [10] is not merely a "bystander phenomenon." The autoantibodies orchestrate a coordinated program of gene expression changes in neuronal cells resulting in decreased neuronal differentiation and decreased neuron survival consistent with the observed acute effects of the autoantibodies in neuroblastoma cells in vitro [10, 18]. These data are the first to suggest that 5-HT2A receptor activation in neuroblastoma cells causes significant downregulation in the $5 \mathrm{ht} 3 \mathrm{~A}$ gene - which encodes a ligand-gated ionic channel important in mediating fast depolarizing responses underlying dopamine release in the striatum [20]. The 5ht3a gene is also expressed in the gut and has a role in intestinal motility [26]. Dysregulating gastrointestinal motility is a common complication in patients suffering with Parkinson's disease.

G-protein coupled receptor kinase 3 (Grk3) was originally reported as the beta- adrenergic receptor kinase-2 (BARK) which phosphorylates the ligand-occupied beta adrenergic receptor leading to beta-arrestin-mediated receptor desensitization [21]. In recent studies, the GRK3 gene isoform was reported to be preferentially highly expressed in olfactory cilia where it functions in desensitization of odorantinduced G-protein coupled receptor signaling [27]. GRK3 knockout mice had altered fast second messenger formation and impaired desensitization following odorant-induced G-protein coupled receptor activation [28]. More study is needed to determine whether downregulation of GRK3 gene by autoantibodies from PD patients may play a role in dysregulated olfaction common in human neurodegenerative disorders [29].

Complex I is the largest of the electron transport chain complexes and is the site of most ROS production. Dysfunction in complex I is closely associated with PD [30]. In an effort to decrease ROS, cells might lower expression of complex I subunits. To increase the flux of electrons down the electron transport chain and thereby limiting the chance for free radical formation, cells may increase expression of ATP synthase subunits. Increase of ATP synthase activity would prevent formation of free radicals by limiting electrons from straying resulting in oxygen molecules with unpaired electron valence shells. Most reactive oxygen species production occurs in the mitochondria and therefore, mitochondrial ROS production would be the main driver of increased cell oxidation responsive genes which include glutathione peroxidase 1 and 4. Parkinsonism associated deglycase (more commonly known as DJ-1 or PARK7) gene expression was only significantly increased in TBI/DM group suggesting small synergistic effect. DJ-1 normal function is to provide neuroprotection, and loss of function mutations in DJ-1 are a known cause of familial PD [31, 32].

The level of 5-HT2A receptor targeting autoantibodies in plasma from either adult obese type 2 diabetes mellitus or TBI alone was previously reported to be significantly associated with systemic inflammation (i.e., WBC) [9]. The present finding that the number of upregulated genes in N2A cells exposed to neurodegenerative autoantibodies was highest in $(\mathrm{TBI}+\mathrm{Db})$ compared to $(\mathrm{Db}$ without $\mathrm{TBI})$ or (TBI alone) may be consistent with chronic inflammation arising from more than one source in $(\mathrm{TBI}+\mathrm{Db})$. In support of this possibility, the mean baseline WBC was nearly significantly higher in (TBI $+\mathrm{Db})$ vs. (Db alone): $12.8 \pm 1.8$ vs $9.2 \pm 0.5 \mathrm{~K} / \mathrm{cm}^{3} ; \mathrm{p}=0.05$. Baseline WBC was more than two-fold higher in (TBI $+\mathrm{Db}$ ) compared to (Db without harm-inducing autoantibodies): $12.8 \pm 1.8$ vs. $6.4 \pm 1.3 \mathrm{~K} /$ $\mathrm{cm}^{3} ; \mathrm{p}=0.015$ ). The clinical relevance of this observation is that lifetime TBI-sufferers are likely to benefit from lifestyle modifications (diet, exercise) that promote maintenance of ideal body weight and lower the risk of future development of obese type 2 diabetes mellitus. In a prior retrospective study, higher body mass index was a significant predictor of the post-TBI occurrence of a composite neurodegenerative disease outcome in eighty middle-aged and older adults [33]. Treatment of type 2 diabetes mellitus which results in long-term improved glycemic control could potentially affect autoantibody levels since it is expected to reduce the risk of microvascular complications. Microvascular diabetic complications were significantly associated with an increased prevalence of harm-inducing 5-HT2AR targeting autoantibodies in an adult type 2 diabetes mellitus population [18].

There are several limitations to the findings in the present study. First, the use of mouse neuroblastoma N2A cells may affect the generalizability of the findings to adult neurons. Future work should consider comparing these responses to those in primary neurons. Second, all study patients were men who suffered from mild TBI. It is not known whether more severe grade of TBI injury or inclusion of women having mild TBI would result in a different pattern or large number of neurodegenerative gene expression changes. 
In summary, RNA seq of neuroblastoma cells exposed to test TBI and T2DM autoantibodies having increased binding to a 5-HT2A receptor peptide involved in receptor activation confirmed involvement of neuron apoptosis in mediating accelerating N2A cell loss downstream of long-lasting receptor activation. It also suggests possible involvement of a number of different genes involved in neurotransmitter release and the regulation of G-protein coupled receptor signaling as candidate mediators of important neurobiological and cardiovascular responses previously associated with increased level of the autoantibodies.

Acknowledgements We thank Drs. Patricia Soteropoulos and Mainul Hoque of the Rutgers-NJMS Genomics Center for expertise with nextgeneration sequencing.

Funding This study was supported by the Biomedical Laboratory Research and Development/Technology Transfer Program and the New Jersey Commission on Brain Injury Research (CBIRPIL007) to MBZ (TTP-001-20S), Assistant Secretary of Defense for Health Affairs through the Congressionally Directed Gulf War Illness Research Program (W81XWH-16-1-0626), the Department of Veterans Affairs (Veterans Health Administration, Office of Research and Development, Rehabilitation/Biomedical Laboratory Research and Development (RX001520, RX003253, BX005015), and the Veterans Bio-Medical Research Institute.

Data Availability All data generated or analyzed during this study are available from the corresponding author on reasonable request. The RNAseq data will be available online in the NCBI GEO.

\section{Declarations}

Ethics Approval and Consent to Participate The study was approved by the VA New Jersey Health Care System Institutional Review Board, conducted in accordance with the ethical standards of the Declaration of Helsinki, and all subjects provided written informed consent prior to participation.

Conflict of interest The authors declare no competing interests.

Disclaimer The contents do not represent the views of the Department of Veterans Affairs or the US Government, and the opinions, interpretations, conclusions, and recommendations are those of the authors and are not necessarily endorsed by the Department of Defense.

Open Access This article is licensed under a Creative Commons Attribution 4.0 International License, which permits use, sharing, adaptation, distribution and reproduction in any medium or format, as long as you give appropriate credit to the original author(s) and the source, provide a link to the Creative Commons licence, and indicate if changes were made. The images or other third party material in this article are included in the article's Creative Commons licence, unless indicated otherwise in a credit line to the material. If material is not included in the article's Creative Commons licence and your intended use is not permitted by statutory regulation or exceeds the permitted use, you will need to obtain permission directly from the copyright holder. To view a copy of this licence, visit http://creativecommons.org/licenses/by/4.0/.

\section{References}

1. Bombardier CH, Fann JR, Temkin NR, Esselman PC, Barber J, Dikmen SS (2010) Rates of major depressive disorder and clinical outcomes following traumatic brain injury. JAMA 303(19):19381945. https://doi.org/10.1001/jama.2010.599

2. Dikmen SS, Corrigan JD, Levin HS, Machamer J, Stiers W, Weisskopf MG (2009) Cognitive outcome following traumatic brain injury. J Head Trauma Rehabil 24(6):430-438. https://doi.org/10. 1097/HTR.0b013e3181c133e9

3. Delic V, Beck KD, Pang KCH, Citron BA (2020) Biological links between traumatic brain injury and Parkinson's disease. Acta Neuropathol Commun 8(1):45. https://doi.org/10.1186/ s40478-020-00924-7

4. Williams DR, Litvan I (2013) Parkinsonian syndromes. Continuum (Minneap Minn) 19 (5 Movement Disorders):1189-1212. https://doi.org/10.1212/01.CON.0000436152.24038.e0

5. Walker KR, Tesco G (2013) Molecular mechanisms of cognitive dysfunction following traumatic brain injury. Front Aging Neurosci 5:29. https://doi.org/10.3389/fnagi.2013.00029

6. Xu T, Pandey SC (2000) Cellular localization of serotonin(2A) (5HT(2A)) receptors in the rat brain. Brain Res Bull 51(6):499505. https://doi.org/10.1016/s0361-9230(99)00278-6

7. Cook EH Jr, Fletcher KE, Wainwright M, Marks N, Yan SY, Leventhal BL (1994) Primary structure of the human platelet serotonin 5-HT2A receptor: identify with frontal cortex serotonin 5-HT2A receptor. J Neurochem 63(2):465-469. https://doi.org/ 10.1046/j.1471-4159.1994.63020465.x

8. Lopez-Gimenez JF, Gonzalez-Maeso J (2018) Hallucinogens and serotonin 5-HT2A receptor-mediated signaling pathways. Curr Top Behav Neurosci 36:45-73. https://doi.org/10.1007/7854 2017_478

9. Zimering MB, Pulikeyil AT, Myers CE, Pang KC (2020) Serotonin 2A Receptor autoantibodies increase in adult traumatic brain injury in association with neurodegeneration. J Endocrinol Diabetes $7(1): 1-8$

10. Zimering MB (2018) Circulating neurotoxic 5-HT2A receptor agonist autoantibodies in adult type 2 diabetes with Parkinson's disease. J Endocrinol Diabetes 5 (2). https://doi.org/10.15226/ 2374-6890/5/2/01102

11. Weber JT (2012) Altered calcium signaling following traumatic brain injury. Front Pharmacol 3:60. https://doi.org/10.3389/fphar. 2012.00060

12. Pivovarova NB, Andrews SB (2010) Calcium-dependent mitochondrial function and dysfunction in neurons. FEBS $\mathrm{J}$ 277(18):3622-3636. https://doi.org/10.1111/j.1742-4658.2010. 07754.x

13. Friel DD (2000) Mitochondria as regulators of stimulus-evoked calcium signals in neurons. Cell Calcium 28(5-6):307-316. https://doi.org/10.1054/ceca.2000.0172

14. Verkhratsky A (2005) Physiology and pathophysiology of the calcium store in the endoplasmic reticulum of neurons. Physiol Rev 85(1):201-279. https://doi.org/10.1152/physrev.00004.2004

15. Gibson GE, Starkov A, Blass JP, Ratan RR (1802) Beal MF (2010) Cause and consequence: mitochondrial dysfunction initiates and propagates neuronal dysfunction, neuronal death and behavioral abnormalities in age-associated neurodegenerative diseases. Biochim Biophys Acta 1:122-134. https://doi.org/10.1016/j.bbadis. 2009.08.010

16. Voss JD, Connolly J, Schwab KA, Scher AI (2015) Update on the epidemiology of concussion/mild traumatic brain injury. Curr Pain Headache Rep 19(7):32. https://doi.org/10.1007/ s11916-015-0506-Z

17. Zimering MB (2017) Diabetes autoantibodies mediate neural- and endothelial cell- inhibitory effects via 5-hydroxytryptamine- 2 
receptor coupled to phospholipase C/inositol triphosphate/Ca2+ pathway. J Endocrinol Diabetes 4 (4). https://doi.org/10.15226/ 2374-6890/4/4/00184

18. Zimering (2019) Autoantibodies in type-2 diabetes having neurovascular complications bind to the second extracellular loop of the 5-hydroxytryptamine 2A receptor. Endocrinol Diabetes Metabol J 3(4): 118

19. Hales TG, Dunlop JI, Deeb TZ, Carland JE, Kelley SP, Lambert JJ, Peters JA (2006) Common determinants of single channel conductance within the large cytoplasmic loop of 5-hydroxytryptamine type 3 and alpha4beta2 nicotinic acetylcholine receptors. J Biol Chem 281(12):8062-8071. https://doi.org/10.1074/ jbc.M513222200

20. Nayak SV, Ronde P, Spier AD, Lummis SC, Nichols RA (2000) Nicotinic receptors co-localize with 5-HT(3) serotonin receptors on striatal nerve terminals. Neuropharmacology 39(13):26812690. https://doi.org/10.1016/s0028-3908(00)00109-x

21. Parruti G, Ambrosini G, Sallese M, De Blasi A (1993) Molecular cloning, functional expression and mRNA analysis of human beta-adrenergic receptor kinase 2. Biochem Biophys Res Commun 190(2):475-481. https://doi.org/10.1006/bbrc.1993.1072

22. Zimering MB, Pan Z (2009) Autoantibodies in type 2 diabetes induce stress fiber formation and apoptosis in endothelial cells. J Clin Endocrinol Metab 94(6):2171-2177. https://doi.org/10.1210/ jc. 2008-2354

23. Chen Y, Neve RL, Liu H (2012) Neddylation dysfunction in Alzheimer's disease. J Cell Mol Med 16(11):2583-2591. https://doi. org/10.1111/j.1582-4934.2012.01604.x

24. DiProspero NA, Chen EY, Charles V, Plomann M, Kordower JH, Tagle DA (2004) Early changes in Huntington's disease patient brains involve alterations in cytoskeletal and synaptic elements. J Neurocytol 33(5):517-533. https://doi.org/10.1007/ s11068-004-0514-8

25. McMahon HT, Missler M, Li C, Sudhof TC (1995) Complexins: cytosolic proteins that regulate SNAP receptor function. Cell 83(1):111-119. https://doi.org/10.1016/0092-8674(95)90239-2

26. Aikiyo S, Kishi K, Kaji N, Mikawa S, Kondo M, Shimada S, Hori $\mathrm{M}$ (2020) Contribution of serotonin 3A receptor to motor function and its expression in the gastrointestinal tract. Digestion:1-11. https://doi.org/10.1159/000509209

27. Schleicher S, Boekhoff I, Arriza J, Lefkowitz RJ, Breer H (1993) A beta-adrenergic receptor kinase-like enzyme is involved in olfactory signal termination. Proc Natl Acad Sci U S A 90(4):1420-1424. https://doi.org/10.1073/pnas.90.4.1420

28. Peppel K, Boekhoff I, McDonald P, Breer H, Caron MG, Lefkowitz RJ (1997) G protein-coupled receptor kinase 3 (GRK3) gene disruption leads to loss of odorant receptor desensitization. J Biol Chem 272(41):25425-25428. https://doi.org/10.1074/jbc.272.41. 25425

29. Dintica CS, Marseglia A, Rizzuto D, Wang R, Seubert J, Arfanakis K, Bennett DA, Xu W (2019) Impaired olfaction is associated with cognitive decline and neurodegeneration in the brain. Neurology 92(7):e700-e709. https://doi.org/10.1212/WNL.0000000000 006919

30. Schapira AH, Cooper JM, Dexter D, Clark JB, Jenner P, Marsden CD (1990) Mitochondrial complex I deficiency in Parkinson's disease. J Neurochem 54(3):823-827. https://doi.org/10.1111/j. 1471-4159.1990.tb02325.x

31. Ariga H, Takahashi-Niki K, Kato I, Maita H, Niki T, Iguchi-Ariga SM (2013) Neuroprotective function of DJ-1 in Parkinson's disease. Oxid Med Cell Longev 2013:683920. https://doi.org/10. $1155 / 2013 / 683920$

32. Repici M, Giorgini F (2019) DJ-1 in Parkinson's disease: clinical insights and therapeutic perspectives. J Clin Med 8 (9). https:// doi.org/10.3390/jcm8091377

33. Zimering MB, Patel D, Bahn G (2019) Type 2 diabetes predicts increased risk of neurodegenerative complications in veterans suffering traumatic brain injury. J Endocrinol Diabetes 6 (3). https:// doi.org/10.15226/2374-6890/6/3/001137

Publisher's Note Springer Nature remains neutral with regard to jurisdictional claims in published maps and institutional affiliations. 